\title{
Morteros, para la impresión 3D con áridos locales de la
}

\section{Zona 3 de Ecuador}

\section{Mortars, for 3D printing with local aggregates from Zone 3 of Ecuador}

Marco Javier Palacios Carvajal. ${ }^{1}$, Oscar Efren Paredes Peñaherrera. ${ }^{2}$ \& Rosa Pamela Taco Hernández. $^{3}$

\begin{abstract}
.
DOI: https://doi.org/10.33262/concienciadigital.v3i2.1.1239

3D printing is a novel process in the construction industry, in several countries studies of different mortars have been carried out for use in 3D printers; however, there is no standardization of materials, equipment and procedures that allow obtaining a mortar that meets all the characteristics to be considered printable; consequently mortars were made with 2 types of aggregates from the province of Chimborazo and Tungurahua, to make a comparison of their physical, chemical and mechanical characteristics.
\end{abstract}

Keywords: 3D printing, mortars, Chimborazo, Tungurahua, aggregates and cements.

\section{Resumen.}

La impresión 3D es un proceso novedoso en la industria de la construcción, en varios países se han realizado estudios de diferentes morteros para su uso en impresoras 3D; sin embargo no existe una estandarización de materiales, equipos y procedimientos que permitan la obtención de un mortero que cumpla con todas las características para ser considerado imprimible; en consecuencia se realizó morteros con 2 tipos de agregados provenientes de la provincia de Chimborazo y Tungurahua, para realizar una comparación de sus características físicas, químicas y mecánicas.

\footnotetext{
${ }^{1}$ Universidad Nacional de Chimborazo, Facultad de Ingeniería. Riobamba, Ecuador.jpalacios@unach.edu.ec

${ }^{2}$ Universidad Nacional de Chimborazo, Facultad de Ingeniería. Riobamba, Ecuador. oeparedes@unach.edu.ec

${ }^{3}$ Universidad Nacional de Chimborazo, Facultad de Ingeniería. Riobamba, Ecuador. rosapamelatacohernandez@gmail.com
} 
Palabras Claves: Impresión 3D, morteros, Chimborazo, Tungurahua, agregados y cementos.

\section{Introducción}

El despunte tecnológico en la industria de la construcción es la impresión 3D; como un sistema que permite la fabricación de elementos de construcción prediseñados con mortero, para el ensamblaje de obras pequeñas en pocas horas, disminuyendo los costos de producción, desperdicios y sistematizando procesos. (Campillo Mejía, 2017)

En el mundo, varios estudios se han desarrollado en busca de un mortero que cumpla las características para considerarse imprimible entre ellas destacan:

Capacidad de ser bombeado.- la consistencia del mortero debe permitir que se mueva por el sistema de la impresora hasta llegar al cabezal de impresión produciendo una masa constante; Torres (2016), definió que para poder ser bombeado el mortero debe ser amasado con un árido fino de tamaño máximo de $8 \mathrm{~mm}$ para evitar taponamientos en el cabezal de impresión.

Capacidad de ser impreso.- la extrucción del mortero por la boquilla de impresión debe ser de tal manera que no se deje restos que puedan obstaculizar la correcta impresión del material; Chimbolema (2017), menciona que el ensayo de trabajabilidad debe obtener un resultado de $150 \pm 10 \mathrm{~mm}$ medida por el ensayo de consistencia en la mesa de sacudidas.

Constructibidad.- se define como la resistencia que tiene el mortero una vez impreso de soportar su peso propio y el de las hiladas superiores sin deformarse excesivamente, Altamirano, Cuevas, \& Sanchez (2015), evaluaron el tiempo de fraguado para el control de dicha característica.

Wu, Wang, \& Wang (2016), mencionaron que las características de un mortero imprimible no solo dependen del agregado fino que se utilice en su amasado, sino también del cemento y aditivos que se utilicen; tanto los aditivos y diferentes tipos de cementos que se usan para la elaboración de un mortero se han estudiado para la obtención de resistencia a la compresión en intervalos de tiempo largos en tanto, que la impresión 3D precisa de tiempos de fraguado cortos y desarrollo de resistencias a tempranas edades, en minutos. (Limonte, 2016)

En Ecuador, existen varias minas que proveen de arena de río como agregado fino cumpliendo varios requisitos que la Agencia de Regulación y Control Minero del país exige, la provincia 
de Chimborazo y Tungurahua son reconocidas como las mejores productoras de arena de río, pues en estas provincias se desempeñan las canteras más grandes. (Mariño, 2018)

\section{Metodología}

Esta investigación inicia con la obtención del agregado fino proveniente de las provincias de Chimborazo y Tungurahua, obteniendo dos muestras de arena de río, diferenciadas por el color de la arena siendo la de Chimborazo más oscura, como es evidente en la figura 1.

Figura 1 Muestras obtenidas para el estudio

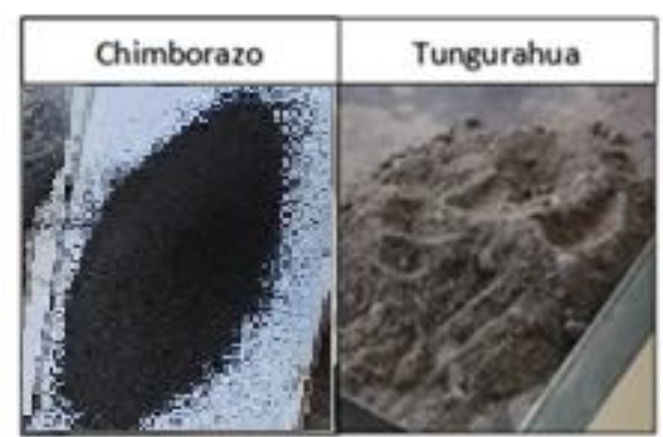

Fuente: Elaboración Propia

El mortero imprimible debe cumplir algunas características antes mencionadas, es por esto que, para asegurar un correcto comportamiento mecánico, se realizó varios ensayos físicos y químicos de los agregados.

Se inició con el ensayo de Contenido de materia orgánica en áridos finos, el cual se desarrolló conforme la norma INEN NTE 0855; el proceso describe que, en un frasco limpio y con tapa se diluye $3 \mathrm{~g}$ de hidróxido de sodio en agua, luego se añade $150 \mathrm{~g}$ de árido fino, mezclar con movimientos de agitación y batido, hasta conseguir que toda la muestre quede totalmente húmeda; dejar reposar por 24 horas, luego de transcurrido este tiempo, se realiza una comparación del tono del color del agua que sobrenada con la tabla de colores de Gardner para su posterior análisis.

Continuamos con el ensayo de granulometría, este ensayo está definido en la norma INEN NTE 696, en su procedimiento define una serie de tamices para áridos finos, por los cuales se deja caer la muestra de árido para luego ser insertado en una tamizadora por el lapso de 10 minutos, 
luego se procede al pesaje del material retenido en cada tamiz para su cálculo y análisis posterior. A continuación, se realizó el ensayo de contenido de humedad siguiendo el proceso: pesamos un recipiente vacío, en este colocamos una porción del árido fino en estado natural y pesamos el conjunto, luego se introduce en un horno de secado a $110{ }^{\circ} \mathrm{C}$ por 24 horas, transcurrido este tiempo dejamos enfriar y pesamos de nuevo el conjunto así lo menciona la norma INEN NTE 690.

Finalmente se realizó el ensayo de densidad aparente y porcentaje de absorción para lo cual; se sumerge una muestra representativa de árido fino en agua por 24 horas para saturar sus partículas, con ayuda del horno de secado llevamos el árido fino hasta el estado de saturado superficialmente seco (SSS) para verificar este estado se llena el molde tronconico y se golpea 25 veces con el martillo del molde para compactar, se retiran los excesos aledaños, luego se levanta el molde con cuidado, si una vez que se retira este cono de arena se desmorona mininamente el árido está en estado SSS; llenamos un picnómetro con agua hasta la marca de calibración y añadimos $500 \mathrm{~g}$ de árido fino en estado SSS y mezclamos hasta conseguir que la muestre este totalmente humedecida, se elimina la mayor cantidad de burbujas y se pesa el conjunto, finalmente tomamos una porción del árido en estado SSS y lo ponemos a secar por 24 horas en un horno de secado a $110^{\circ} \mathrm{C}$ para posteriormente pesar la muestra, este proceso lo describe la norma INEN NTE 857.

Los cementos utilizados en esta investigación se los obtuvo en la Unión Cementera Nacional, obteniendo datos muy importancia y de relevancia para la investigación; de igual manera se decidió la utilización de un aditivo plastificante-acelerante para el amasado del mortero pues en la impresión 3D se necesita el desarrollo de resistencia en intervalos de tiempo de fraguado cortos y los cementos utilizados han sido desarrollados para tiempos de fraguados largos.

Dopico (2008), menciono que la cal hidráulica añadida en un mortero durante su amasado incrementa la resistencia a la compresión por que las partículas de dicha cal actúan como absorbente de agua ayudando al endurecimiento de la mezcla, por ello en esta investigación se utilizó cal hidráulica de la zona 3 del país.

Una vez conocidos todos estos datos, se realizó una elección de dosificaciones para evaluar el comportamiento mecánico de los áridos finos en el mortero, las cuales se muestran en la tabla No 1. 
Tabla 1 Descripción de dosificaciones

\begin{tabular}{lcccc}
\hline Componentes & M-0-I & M-7-I & M-0-HE & M-7-HE \\
\hline Árido Fino (Kg) & 62.25 & 67.20 & 62.25 & 67.20 \\
Cemento (Kg) & 50.00 & 50.00 & 50.00 & 50.00 \\
Cal (Kg) & 0.00 & 3.50 & 0.00 & 3.50 \\
Agua (Kg) & 21.00 & 25.45 & 20.75 & 22.45 \\
Plastificante (Kg) & 1.00 & 1.00 & 1.00 & 1.00 \\
\hline
\end{tabular}

Elaborado por: grupo de investigación

La nomenclatura de los morteros está dada por la letra (M) de mortero, seguido del porcentaje de cal hidráulica en la dosificación (0 ó 7) y a continuación el tipo de cemento utilizado para el amasado del mortero ( $\mathrm{I}$ - HE); las dosificaciones fueron realizados en igual cantidad de los componentes para los dos tipos de áridos mencionados anteriormente, para poder realizar una comparación más representativa del comportamiento mecánico de la arena de río en el mortero. La Norma Ecuatoriana de la Construcción (NEC, 2010), menciona el proceso de amasado para un mortero; en el cual describe que el árido fino, cemento y en este caso la cal hidráulica serán mezclados hasta obtener una color homogéneo, a continuación se añade el aditivo y se bate para finalmente agregar el agua y continuar con el batido de estos elementos; hasta obtener una mezcla homogénea, cuidando la adición involuntaria de materiales orgánicos e inorgánicos en el mortero.

Una vez realizado el amasado del mortero se procedió con algunos ensayos mecánicos para definir el cumplimiento de las propiedades de los morteros para considerarlos imprimibles.

Según la norma INEN NTE 488, se debe fabricar probetas de ensayo cúbicas de (50*50) mm; para esto se fabricaron moldes de madera y para evitar que el mortero se adhiera al molde se sumergió este por 24 horas en diésel, estos moldes se pueden utilizar como máximo 2 veces. Para el ensayo de resistencia a la compresión de morteros se fabricaron 4 probetas cúbicas por edad de ensayo y se realizó el procedimiento de la norma INEN NTE 488; la probeta cubica se coloca en el centro del pistón hidráulico de la prensa electrónica para asegurar una distribución de carga uniforme en todo el cuerpo de la probeta hasta llegar a su ruptura; los datos obtenidos serán tabulados para su posterior calculo y análisis. 
Además, se realizó el ensayo de tiempo de fraguado del mortero basado en la norma INEN NTE 158, para lo cual se utilizó un molde de PVC circular de $5 \mathrm{~cm}$ de altura, en este molde se vierte el mortero y mediante el ensayo de Vicat se determinó el tiempo de inicio de fraguado y el tiempo final de fraguado.

El ensayo de trabajabilidad se desarrolló elaborando rollos de cada mortero los cuales fueron colocados directamente en la mesa de sacudidas, este ensayo se determina hasta obtener el menor diámetro posible sin que el rollo se vea fracturado o roto, esto se realizó basado en la norma INEN NTE 157, para la determinación de la consistencia del mortero.

\section{Resultados}

Según lo descrito en el capítulo anterior se realiza un contraste de resultados obtenidos por los áridos finos en los ensayos físicos y químicos en iguales condiciones.

Contenido orgánico en agregado fino. - Esta comparación se realizó a las 24 horas de realizado el ensayo, los resultados se muestran en la figura 2.

Figura 2 Comparación de los resultados de las muestras con la Tabla de Gardner

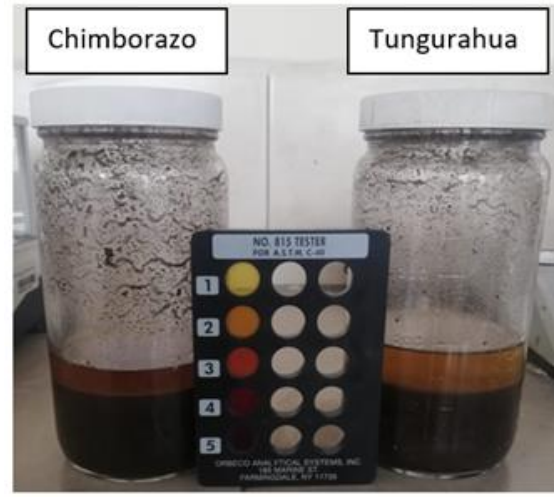

Fuente: Elaboración Propia

La arena de río de la provincia de Chimborazo tiene un alto contenido de materia orgánica a comparación del agregado proveniente de la provincia de Tungurahua; lo cual es perjudicial para un mortero imprimible, por tanto, con el tiempo estas impurezas se reproducen creando fisuras que afectan al comportamiento mecánico del mortero.

Granulometría. - A continuación, la figura 3, indica las curvas granulométricas de los áridos finos evidenciando que cada una de estas se encuentran dentro de los límites establecidos, así 
también, así se determina que sus módulos de finura están dentro del rango establecidos los mismos que van de 2.3 a 3.1 como lo indica la NEC (2010).

Figura 3 Curvas granulométricas
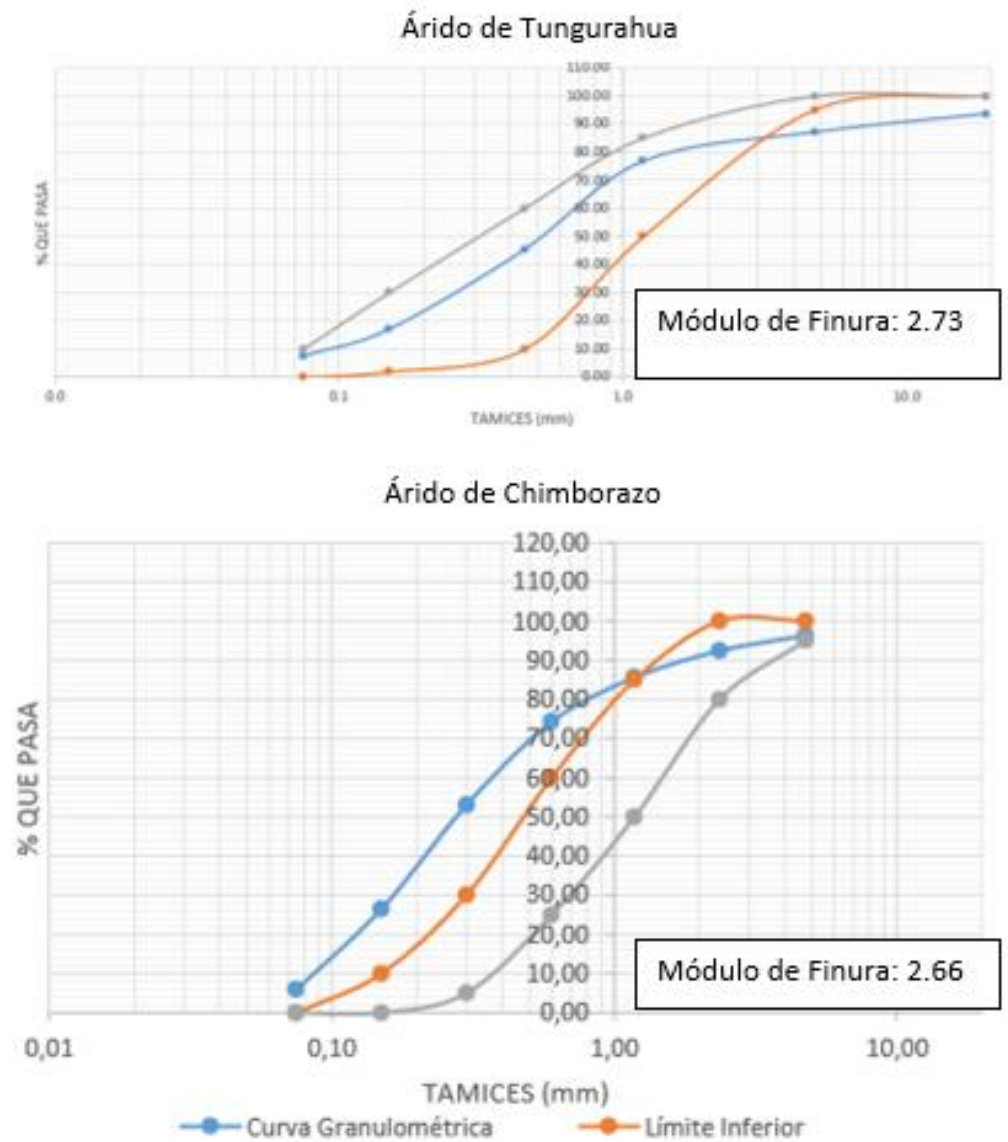

Fuente: Elaboración Propia

De los resultados, los módulos de finura se encuentran dentro de los rangos establecidos lo que significa, que en el amasado del mortero las partículas podrán ser extruidas por el cabezal de la impresora 3D sin producir taponamientos y de manera continua su impresión.

Contenido de humedad. - En la tabla 2 se muestran los resultados del ensayo, obteniendo resultados dentro de los parámetros para un mortero, que indica que no debe superar el 10\% para evitar una mezcla muy suelta ni menor a $4 \%$ para evitar mezclas secas. 
ISSN: 2600-5859

Tabla 2 Contenido de humedad

\begin{tabular}{|c|c|c|c|c|}
\hline \multicolumn{5}{|c|}{ ÁRIDO DE TUNGURAHUA } \\
\hline Recipientes & $\begin{array}{l}\text { Masa Rec } \\
(\mathrm{g})\end{array}$ & $\begin{array}{c}\text { Masa Rec + Muestra } \\
\text { húmeda }(\mathrm{g})\end{array}$ & $\begin{array}{c}\text { Masa } \\
\text { Rec+Muestra } \\
\text { seca }(\mathrm{g})\end{array}$ & $\begin{array}{c}\% \\
\text { Humedad }\end{array}$ \\
\hline Tara 1 & 18.400 & 82.900 & 77.500 & 9.137 \\
\hline Tara 2 & 18.000 & 92.400 & 86.300 & 8.931 \\
\hline & & & $\begin{array}{c}\% \text { Humedad } \\
\text { Promedio } \\
\end{array}$ & 9.03 \\
\hline \multicolumn{5}{|c|}{ ÁRIDO DE CHIMBORAZO } \\
\hline Recipientes & $\begin{array}{l}\text { Masa Rec } \\
(\mathrm{g})\end{array}$ & $\begin{array}{c}\text { Masa Rec + Muestra } \\
\text { húmeda }(\mathrm{g})\end{array}$ & $\begin{array}{c}\text { Masa } \\
\text { Rec+Muestra } \\
\text { seca }(\mathrm{g}) \\
\end{array}$ & $\begin{array}{c}\% \\
\text { Humedad }\end{array}$ \\
\hline Tara 1 & 16.690 & 99.820 & 95.540 & 5.428 \\
\hline \multirow[t]{2}{*}{ Tara 2} & 17.030 & 106.560 & 101.460 & 6.041 \\
\hline & & & $\begin{array}{c}\% \text { Humedad } \\
\text { Promedio }\end{array}$ & 5.73 \\
\hline
\end{tabular}

Elaborado por: grupo de investigación

Los porcentajes de humedad de los dos áridos en estudio están dentro de los límites establecidos por la normativa, lo cual nos asegura que para las dosificaciones planteadas tendremos mezclas homogéneas con una trabajabilidad adecuada y que se controlara de mejor manera la relación agua-cemento.

Densidad aparente y porcentaje de absorción. - Los resultados del ensayo se muestran en la tabla 3, los cuales cumplen con lo establecido en la norma para considerar un árido de calidad.

Tabla 3 Porcentaje de absorción.

\begin{tabular}{ccc}
\hline Valores & Tungurahua & Chimborazo \\
\hline Densidad $(\mathrm{kg} / \mathrm{m} 3)$ & 2072.73 & 1494.18 \\
Densidad en SSS $(\mathrm{kg} / \mathrm{m} 3)$ & 2159.09 & 1589.38 \\
Densidad Aparente $(\mathrm{kg} / \mathrm{m} 3)$ & 2269.19 & 1651.82 \\
\% de Absorción & 4.17 & 6.37 \\
\hline
\end{tabular}

Elaborado por: grupo de investigación

El porcentaje de absorción del árido de Chimborazo es mayor que el de Tungurahua lo que significa que este árido absorbe humedad en mayor cantidad, el mismo afecta la relación agua- 
cemento en el mortero lo cual es desfavorable para el amasado; por ello se utilizó el aditivo antes mencionado.

A continuación de estos ensayos se evaluaron las características mecanicas de estos morteros y los resultados se presentan a continuación.

Resistencia a la compresión a corto plazo. - Los resultados de este ensayo se muestran en la tabla 4, siendo el mortero con cemento tipo HE sin cal hidráulica y con agregado de la provincia de Chimborazo el que mejor resistencia a la compresión presenta.

Tabla 4 Resistencia a la compresión a corto plazo

\begin{tabular}{ccccc}
\hline Provincia & Dosificación & 6 Horas & 9 Horas & 24 Horas \\
\hline Tungurahua & M-0-I & 7.81 & 11.20 & 19.65 \\
Compresión & M-7-I & 5.83 & 9.08 & 15.47 \\
(Mpa) & M-0-HE & 7.89 & 16.19 & 20.52 \\
& M-7-HE & 5.92 & 13.26 & 16.57 \\
\hline \multirow{2}{*}{ Chimborazo } & M-0-I & 4.08 & 7.60 & 12.75 \\
Compresión & M-7-I & 3.08 & 4.04 & 11.98 \\
(Mpa) & M-0-HE & 10.09 & 17.48 & 23.85 \\
& M-7-HE & 8.992 & 17.608 & 21.132 \\
\hline
\end{tabular}

Elaborado por: grupo de investigación

Figura 4 Resistencia a la compresión a corto plazo vs. Tiempo

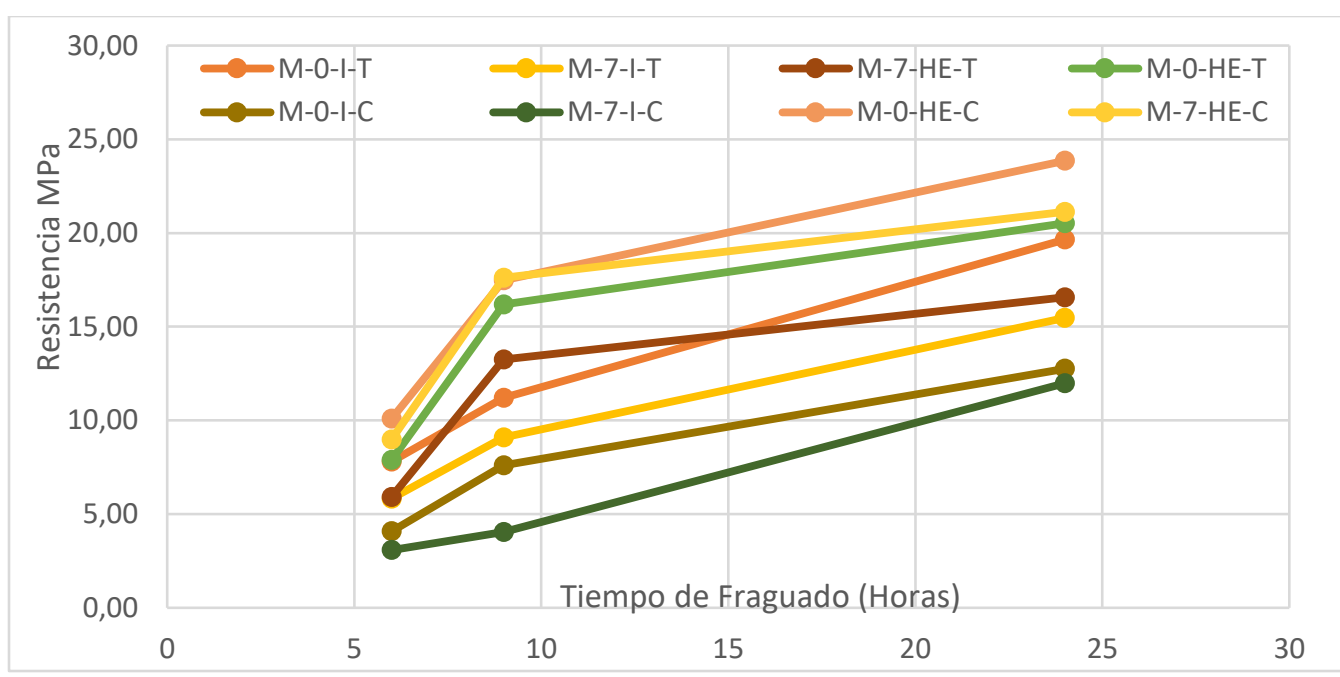

Fuente: Elaboración propia

Como se observa en la figura 4, los morteros que contienen cal hidráulica presentan un desarrollo de resistencia mucho menor a los que no tienen cal, esto quiere decir que la cal hidráulica no está aumentando la resistencia como lo menciono Dopico (2008) en nuestro caso. 
Resistencia a la compresión a largo plazo. - Los resultados del ensayo en mención se muestran en la tabla 5, donde se videncia que el mortero con cemento HE sin añadidura de cal y con árido de la provincia de Chimborazo desarrollo mayor resistencia, además notamos que la resistencia de su similar con cal hidráulica también tiene buen resultado.

Tabla 5 Resistencia a la compresión a largo plazo

\begin{tabular}{cccccc}
\hline Provincia & Dosificación & 7 Días & 14 Días & 21 Días & 28 Días \\
\hline \multirow{2}{*}{ Tungurahua } & M-0-I & 30.09 & 38.12 & 43.68 & 44.72 \\
Compresión & M-7-I & 24.17 & 29.44 & 31.96 & 36.40 \\
(Mpa) & M-0-HE & 36.28 & 43.16 & 45.84 & 48.24 \\
& M-7-HE & 27.84 & 33.56 & 38.72 & 42.24 \\
\hline \multirow{2}{*}{ Chimborazo } & M-0-I & 28.13 & 36.17 & 40.71 & 41.11 \\
Compresión & M-7-I & 19.84 & 25.99 & 29.23 & 30.49 \\
(Mpa) & M-0-HE & 50.18 & 58.57 & 62.14 & 63.82 \\
& M-7-HE & 41.72 & 47.62 & 56.05 & 57.75 \\
\hline
\end{tabular}

Elaborado por: grupo de investigación

Figura 5 Resistencia a la compresión a largo plazo vs. Tiempo

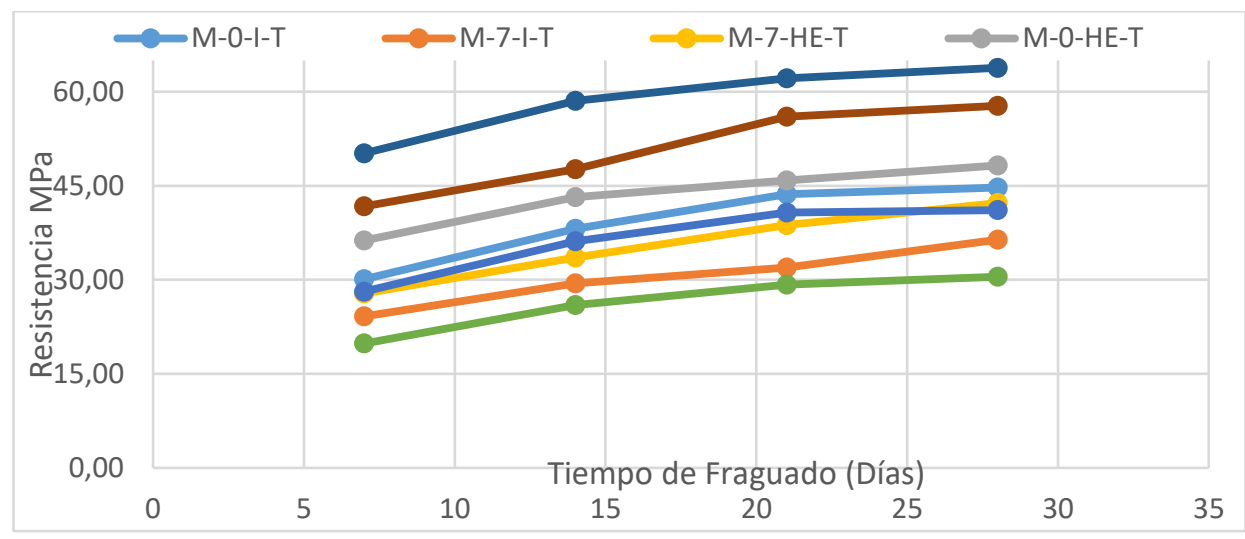

Fuente: Elaboración propia

De la figura 5, se evidencia que los desarrollos de resistencias a largo plazo siguen un patrón definido, sin embargo, los morteros elaborados con cemento HE se desarrollan de mejor manera, así mismo se puede observar que la cal hidráulica no ayuda al desarrollo de resistencia. Por tanto, se desecha la hipótesis de la utilización de la cal hidráulica para fortalecer la resistencia de los morteros. 
Es importante mencionar que todos los resultados alcanzados en las características de resistencia superan el valor común de un hormigón que es de $21 \mathrm{MPa}$, lo cual es una ventaja que debe ser aprovechada para la utilización de estos morteros en otras aplicaciones constructivas para beneficio de la industria de la construcción.

Trabajabilidad. - los resultados del ensayo se resumen en la tabla 6, donde se comparan los morteros con los dos tipos de áridos.

Tabla 6 Diámetros establecidos para evaluar la trabajabilidad.

\begin{tabular}{cccccc}
\hline Morteros & Minutos & $\mathbf{1}$ & $\mathbf{2}$ & $\mathbf{3}$ & $\mathbf{5}$ \\
\hline \multirow{2}{*}{ M-0-I } & Chimborazo & $21 \mathrm{~cm}$ & $19.5 \mathrm{~cm}$ & $19 \mathrm{~cm}$ & $16.5 \mathrm{~cm}$ \\
& Tungurahua & $22 \mathrm{~cm}$ & $20 \mathrm{~cm}$ & $19.75 \mathrm{~cm}$ & $14 \mathrm{~cm}$ \\
\hline \multirow{2}{*}{ M-7-I } & Chimborazo & $18 \mathrm{~cm}$ & $16 \mathrm{~cm}$ & $15 \mathrm{~cm}$ & $14.8 \mathrm{~cm}$ \\
& Tungurahua & $17 \mathrm{~cm}$ & $15 \mathrm{~cm}$ & $14.5 \mathrm{~cm}$ & $14.02 \mathrm{~cm}$ \\
\hline \multirow{2}{*}{ M-0-HE } & Chimborazo & $22 \mathrm{~cm}$ & $19.5 \mathrm{~cm}$ & $18 \mathrm{~cm}$ & $16 \mathrm{~cm}$ \\
& Tungurahua & $21 \mathrm{~cm}$ & $20.5 \mathrm{~cm}$ & $19.5 \mathrm{~cm}$ & $17 \mathrm{~cm}$ \\
\hline \multirow{2}{*}{ M-7-HE } & Chimborazo & $19 \mathrm{~cm}$ & $16.5 \mathrm{~cm}$ & $15.5 \mathrm{~cm}$ & $14.3 \mathrm{~cm}$ \\
& Tungurahua & $22 \mathrm{~cm}$ & $21.5 \mathrm{~cm}$ & $20.4 \mathrm{~cm}$ & $19.5 \mathrm{~cm}$ \\
\hline
\end{tabular}

Elaborado por: grupo de investigación

Los diámetros de trabajabilidad son medidos en centímetros como ilustra la tabla 6, estos tienen una tendencia de decreciente en el tiempo, lo que significa que no hay escurrimiento y las mezclas se estabilizan de forma inmediata.

Es notorio que el mortero M-0-HE con árido de la provincia de Chimborazo inicia con un diámetro de $22 \mathrm{~cm}$ y llega a uno de $16 \mathrm{~cm}$ cumpliendo con lo establecido en la norma de diámetro inicial y diámetro final, esta característica de trabajabilidad es de importancia en el proceso de impresión 3D pues de esta se desprende el antes, durante y después del proceso de extracción del mortero; las otras mezclas cumplen en cierto porcentaje de la característica de trabajabilidad sin embargo pueden crear taponamientos en el cabezal de impresión.

\section{Discusión}

Los resultados de todos los ensayos antes mencionados de los áridos finos, se define que la característica del agregado fino de la provincia de Chimborazo presenta los mejores resultados que las normativas vigentes así lo requieren; sin embargo, tampoco se puede desechar el árido fino de la provincia de Tungurahua debido a que sus características son semejantes, y además cumpliendo con la normativa en menor proporción. 


\section{Conclusiones}

- El árido fino de la provincia de Chimborazo amasado con cemento tipo HE presenta mejor características de resistencias, trabajabilidad y tiempos de fraguado frente a los morteros amasados con árido de río de la provincia de Tungurahua. Los dos áridos presentan diferencias significativas en cuanto a propiedades físicas, siendo evaluados con normativa vigente que no se ha estudiado enfocada en su utilización en impresoras 3D, verificando las características establecidas por Khalil (2017) para definir un mortero como imprimible.

- Así también analizados los valores de resistencia a la compresión a largo y corto plazo se notó el decrecimiento representativo de los morteros amasados con cal hidráulica respecto a los que no contienen cal hidráulica en su dosificación; siendo más evidente estos decrecimientos por los tipos de cementos utilizados en la investigación. Es por esto que se denota necesario el desarrollo de cementos y aditivos que se utilicen netamente en la obtención de morteros para impresoras 3D.

- Los morteros evaluados con dos tipos de arena de río como agregado fino poseen resultados en el ensayo de trabajabilidad muy alentadores sin embargo es necesario realizar una simulación de impresión para verificar que los morteros no ocasionen taponamientos en el cabezal de impresión.

\section{Referencias}

Altamirano, G., Cuevas, A., \& Sánchez, Z. (2015). Utilización De Fibra De Coco En La Elaboración De Piezas Ecosustentables De Mampostería. Perspectiva En México Y Avance De Estudio. Congreso Nacional de Ingeniería Sísmica, (January 2016).

Retrieved from https://www.researchgate.net/publication/288991375_Utilizacion_de_Fibra_de_Coco_en _la_Elaboracion_de_Piezas_Ecosustentables_de_Mamposteria_Perspectiva_en_Mexico_ y_Avance_de_Estudio

Campillo Mejía, M. (2017). "Prefabricación en la arquitectura: Impresión 3D en hormigón." Universidad Politécnica de Madrid, 40. Retrieved from http://oa.upm.es/47556/

Chimbolema, S. (2017). Diseño de un Mortero con Arcillas y Cemento Portland Tipo I para su uso en Impresoras 3D. Universidad Nacional de Chimborazo, 53. Retrieved from http://dspace.unach.edu.ec/handle/51000/4341

Dopico, J. J., Hernández, F. M., Day, R. L., Middendorf, B., Gehrke, M., \& Martinez, L. 
(2008). Desarrollo de hormigones con aglomerante cal-puzolana fina como material cementicio suplementario. Revista Ingeniería de Construcción, 23(3), 171-178.

INEN NTE 0855. (2010). Determinación de impurezas orgánicas en el árido fino para hormigón. Ecuador, 0855.

INEN NTE 157. (2010). Determinación de la consistencia normal. Método Vicat. Ecuador.

INEN NTE 158. (2010). Determinación del tiempo de fraguado, Método VICAT. Ecuador.

INEN NTE 488. (2010). Determinación de la resistencia a la compresión de morteros en cubos de 50mm de arista. Ecuador.

INEN NTE 690. (2010). Determinación del contenido de agua método del secado al horno. Ecuador.

INEN NTE 696. (2010). Análisis Granulométrico en los áridos, fino y gruesos. Ecuador.

INEN NTE 857. (2010). Determinación de la densidad, densidad relativa y absorcion del árido grueso. Ecuador.

Khalil, N., Aouad, G., El Cheikh, K., \& Rémond, S. (2017). Use of calcium sulfoaluminate cements for setting control of 3D-printing mortars. Construction and Building Materials, 157, 382-391. https://doi.org/10.1016/j.conbuildmat.2017.09.109

Limonte Morales Giselle. (2016). Evaluación de medidas de mitigación de la fisuración debido a la retracción por secado en hormigones para obras hoteleras en Cayo Coco. Universidad Central Martha Abreu.

Mariño Salguero, C. E. (2018). Diseño de un mortero con cemento portland tipo I y HE incluida cal hidráulica para su uso en impresoras 3D. Universidad Nacional de Chimborazo.

NEC. (2010). Norma Ecuatoriana de la Construcción. Ecuador.

Torres Remón, R. (2016). Diseño de hormigón para impresión en 3D. Universidad Politécnica de Valencia, 83.

Wu, P., Wang, J., \& Wang, X. (2016). A critical review of the use of 3-D printing in the construction industry. Automation in Construction, 68, 21-31. https://doi.org/10.1016/j.autcon.2016.04.005 
Alaminos-Fernández, A. F. (2014). Cuando las letras son música: las canciones en inglés en la publicidad española.

Beltrán, M. (2017). El aprendizaje del idioma inglés como lengua extranjera. Revista Boletín Redipe, 6(4), 91-98.

Bernal Vázquez, J. E. (2010). La Música en la enseñanza-aprendizaje del Inglés.

Bernal, C. (2017). Uso de las TICS y su influencia con la enseñanza-aprendizaje del idioma inglés en los estudiantes del I y II ciclo de la Escuela Académico Profesional de la Facultad de Educación UNMSM-Lima . Doctoral dissertation, UNIVERSIDAD NACI.

Bustos Flores, P. F. (2012). El uso de materiales audiovisuales y su influencia en el aprendizaje del idioma inglés.

Castro Martínez, N. D. (2014). El uso de la Música para la enseñanza del Inglés. El Lipdub.

Darian, S. (1969). Antecedentes de la enseñanza moderna de idiomas: Sweet, Jespersen y Palmer. The Modern Language Journal, 53 (8), 545-550.

Díaz, C. M. (2010). Una fotografía de las cogniciones de un grupo de docentes de inglés de secundaria acerca de la enseñanza y aprendizaje del idioma en establecimientos educacionales públicos de Chile. Folios, 31.

Escobar, E. Y. (2005). La música pop en el desarrollo de las habilidades de escucha y producción oral en inglés. Lenguaje. 33, 159-195.

García-Medall Villanueva, J. A. (2001). La traducción en la enseñanza de lenguas.

Medina, M. M. (2013). La importancia del aprendizaje del idioma inglés a temprana edad. Yachana Revista Científica, 2.

Pérez Morales, J. I. (2008). La evaluación como instrumento de mejora de la calidad del aprendizaje. Propuesta de intervención psicopedagógica para el aprendizaje del idioma inglés.

Sotelo, E. R. (2011). Implementación de una metodología de aprendizaje del idioma inglés basado en la música como recurso didáctico. Bachelor's thesis, Universidad Libre.

Tomatis, A. (. (1969). El oído y el lenguaje . Martínez Roca.

\section{【 Ciencia}




\section{PARA CITAR EL ARTÍCULO INDEXADO.}

Palacios Carvajal, M. J., Paredes Peñaherrera, O. E., \& Taco Hernández, R. P. (2020). Morteros, para la impresión 3D con áridos locales de la Zona 3 de Ecuador. ConcienciaDigital, 3(2.1), 250-264. https://doi.org/10.33262/concienciadigital.v3i2.1.1239

\section{\Ciencia}

El artículo que se publica es de exclusiva responsabilidad de los autores y no necesariamente reflejan el pensamiento de la Revista Conciencia Digital.

El artículo queda en propiedad de la revista y, por tanto, su publicación parcial y/o total en otro medio tiene que ser autorizado por el director de la Revista Conciencia Digital.

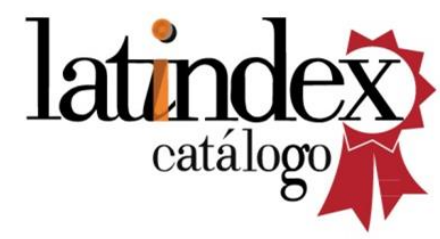

